\title{
ADAPTATION OF REMOTE PRACTICAL WORKS WITH SMART ELECTRONIC PLATFORM BASED ON ARTIFICIAL INTELLIGENCE
}

\author{
Abdelali El Gourari ${ }^{1 *}$, Mustapha Raoufi ${ }^{2}$, and Mohammed Skouri ${ }^{1}$ \\ ${ }^{1}$ Laboratory of Physics High Energy and Astrophysics, Faculty of Science Semlalia, Cadi Ayyad University, Marrackech, Morocco \\ ${ }^{2}$ Laboratory of Materials Energy and Environment, Faculty of Sciences Semlalia, Cadi Ayyad University, Marrakech, Morocco
}

KEYWORDS: Distance learning, intelligent platform, Artificial intelligence algorithms, Remote practical works

\begin{abstract}
:
Distance learning is part of the educational process and has received little attention in current scientific research. However, this type of learning is fundamental in scientific and technical disciplines. It can meet real needs and complement or compensate for classes by a range of advantages such as ensuring the mobility and overcrowding of students and teachers and maintaining a healthy atmosphere within the faculty, saving time and effort. We will have the opportunity to work with many students simultaneously, develop their skills and improve their ability to work in several ways. This paper aims to adapt the remote practical work with an intelligent electronic platform using artificial intelligence techniques for the success and continuation of the educational process. Additionally, we will measure how students interact with an intelligent platform that we have created. This platform has been developed with a software background of LabVIEW, PHP, HTML, CSS, Python, and some artificial intelligence algorithms capable of managing and controlling remote practical work done by students.
\end{abstract}

\section{INTRODUCTION AND RELATED WORK}

In recent utensils, significantly since the beginning of the Corona crisis, the world has changed dramatically. Many problems have been created in various economic, political, and social spheres, and everyone is racing to find alternative solutions to these problems. Given our interest in education and the development of intelligent teaching methods, we have faced many challenges during this crisis; we must take reasonable control of the situation by developing a set of strategies in line with the requirements of students, professors, and administrators. These requirements have led us to consider creating and developing an intelligent electronic platform capable of clever and excellent organization and control of remote practical works (RPWs) to ensure educational continuity. In addition, one of the advantages of this platform is that it can store and analyse all data taken from students and professors during access. This data and analysis helped us cultivate a system based on artificial intelligence technologies found in the paper (El Gourari et al., 2021) that helps guide and assist students in an intelligent way based on their leave and skills in handling electronic circuits. There is a lot of related work in this path (Petrenko et al., 2020) illustrate the cleared concepts' system in the context of their stated research theme, their current state of skilled workers' and junior specialists' distance education in the Ukrainian vocational education and training institutions. (Karadeniz, 2009) create an electronic learning environment that allows learners to access information anywhere, including the distance learning concept. (Lenar et al., 2014) gives an analysis of forms of learning, offers main directions of development of distance learning, and shows differences of distance learning from the traditional.

Distance learning is a new concept in the educational process. Unlike the traditional education system, it does not depend on the classroom and the direct attendance of students with the teacher at the same time and place. Also, it is based on the use of different technological techniques and the design of an integrated education system. In addition, distance learning is a qualitative shift in the educational process. It is also a means by which the information received by students from teachers is preserved through the registration and preservation of lectures (Liang et al., 2017). These are a lot of objectives of distance learning: The aim of establishing the e-learning system is to facilitate and strengthen the traditional process of education to develop it and achieve its objectives. Many of the purposes of installing the distance learning system have been set up to ensure its effectiveness through the achievement of these goals. Precise targeting contributes to facilitating the achievement of these goals. The new design of educations' objectives focuses on developing the educational process and following methods for creating communication between teachers and students.

Our work aims to create a modal of all existential practical works procedures through RPWs in our innovative platform. We will address this paper in two parts. Part one is a valuable framework that summarizes all the practices followed for conducting the RPW of a linear passive quadrupole. Part two will be practical, where we will present a real circuit simulator using the NI ELVIS II+ board and the LABVIEW software to get a virtual circuit linked to the actual course already achieved on this board. After completing the time limit for RPW, the platform stores and processes all student data. Based on artificial intelligence algorithms, the platform evaluates and classifies students by their levels and profile. The platform gives feedback to the teacher to know what students need to improve their grades and abilities.

\section{MATERIALS USED IN THIS STUDY}

\subsection{NI ELVIS II+}

A device provides an integrated set of 12 devices most common in laboratories, including (Oscilloscope, digital multivariate, function generator, variable power supply, and bodied analyser...) as shown in figure 1 . It can also be connected to the computer to make different measurements after building the model on the board. Physics students usually learn the theory of electronic devices and drawing circuits. This board allows these students to implement these concepts. For example, physics students can use its series to create signal conditioning circuits for standard sensors like photovoltaic complications or light detector sensors. 


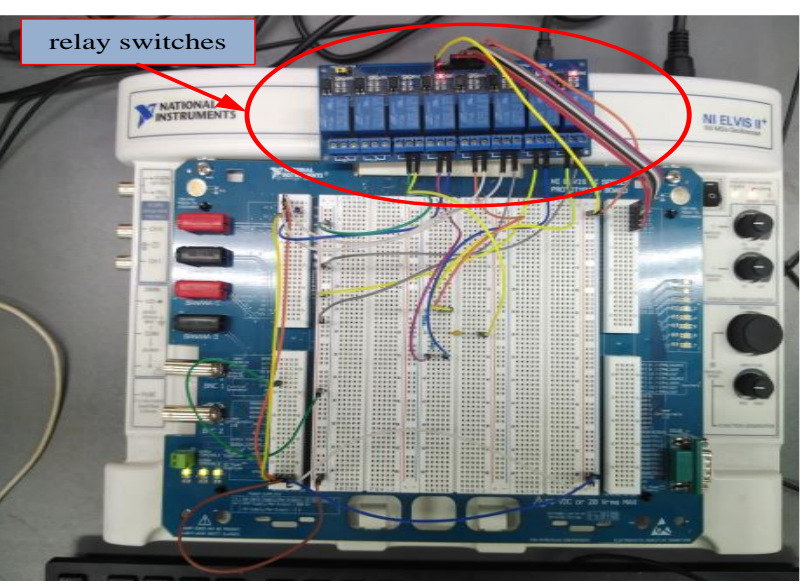

Figure 1. NI ELVIS II+ board with relay switches.

Additionally, the NI ELVIS II+ board is the model on which we will build our natural circle, using the built-in functions to make different measurements and simulating the circuit with the program LabVIEW, which will connect to the actual circuit. These connections will allow us to do RPW to secure the practical simulated component to the essential elements achieved on the board with switches to access precautionary measures.

\subsection{LabVIEW}

LabVIEW is a development environment specializing in industrial and scientific computing. Its specificity is based on the $\mathrm{G}$ language, established by national instruments, which is fully graphic. It allows for the creation of complex software while facilitating programming and thus reducing development time. Due to its functional libraries dedicated to data acquisition, hardware, and mathematical analysis of measurements and the rapid establishment of high-quality graphical interfaces and simplified coding, the engineer has more time to focus on the commercial functions of the hardware and measurement processing. LabVIEW can also simulate processes of all kinds (videos, electronics, mathematics, sounds). This environment includes two windows:
- Front panel allows you to see what the operator might see if the system is manufactured (for example, Oscilloscope with its buttons, blending schedule...

- Block diagram that you can program your system.

\subsection{HTML, CSS, PHP, JavaScript and Python}

Suppose you decide to create your website at the lowest possible cost. In that case, you should be familiar with several programming languages and web design, such as Hypertext Mark-up Language (HTML), Syntax Page Language Cascading Style Sheets (CSS), and Hypertext Pre-processor (PHP). To receive and transmit student and teacher data to the server, MySQL manages our relational dataset. Also, control the JavaScript platform dynamics. To assign artificial intelligence algorithms to the platform, we relied on Python and have graffiti design skills and some other skills. Multiple platforms and locations depend on the services they provide, so during this report, we will try to review essential services, focusing on the advantages they provide so that everyone at any level can handle them easily.

\subsection{Artificial intelligence}

The artificial intelligence that we see now is the science of algorithms. It is most refined and complete form has added to this intelligences' digital and dynamic nature, which provides a different field that cannot be found in the traditional environment typical of school today. Artificial intelligence applications in education (El Gourari et al., 2020) will enable the discovery of new learning boundaries and accelerate the creation of innovative technologies.

\section{PROPOSED WORK}

As we pointed out previously, our goal is to develop an intelligent platform that can adapt quickly and flexibly to students to do RPW in an atmosphere of desire and enthusiasm, so in figure 2 we have proposed an illustration of the course of the work done.

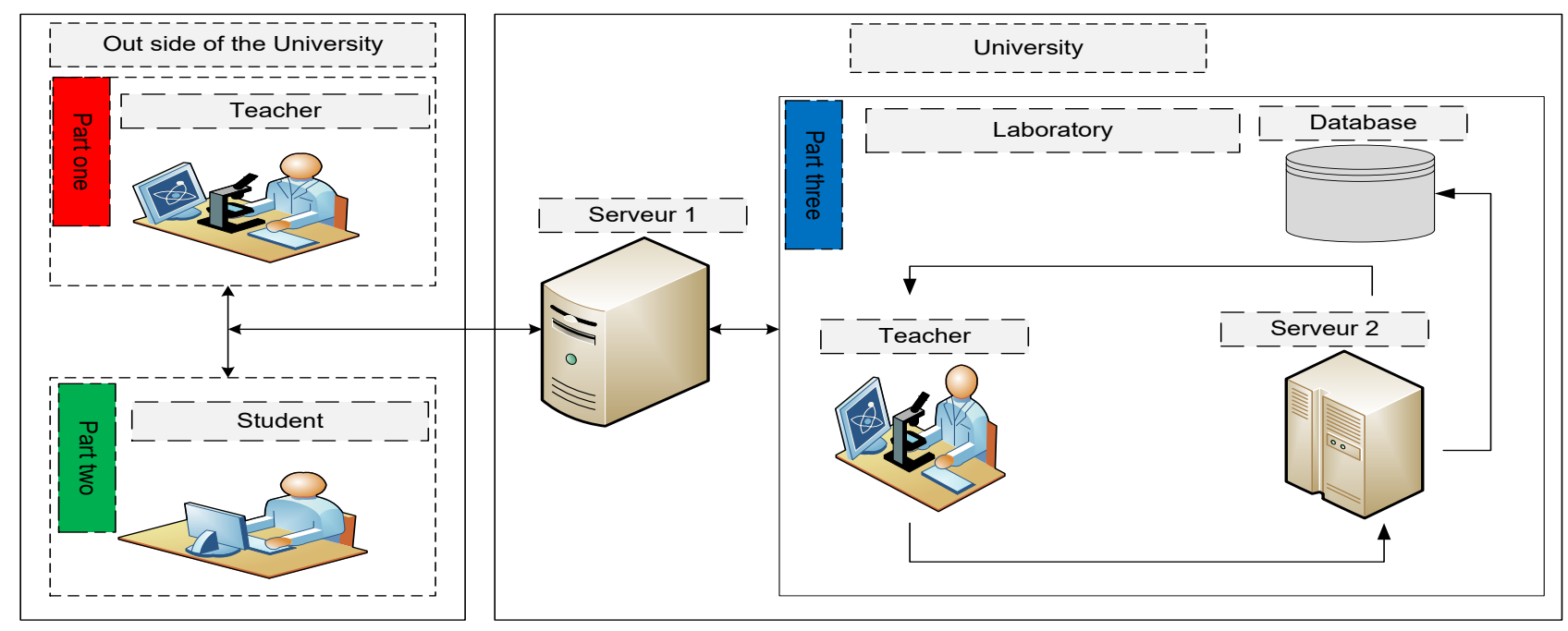

Figure 2. The architecture of the proposed work 


\subsection{Part One}

The professor shall be the manager of all completed and presented to students, whose work shall be as follows:

- To have an account on the platform in advance

- Enter this account into the platform and divide the students into groups, after which a period is assigned to each group.

- Delete or add new students.

- The process to be carried out by students is prepared by the LabVIEW environment as illustrated in figure 1 and coordinated with the NI ELVIS II+ card. This card enables us to complete and control operations through the LabVIEW program; in other words, any action done is simulated with the LabVIEW program and this card.

- Recording all that the professor has done by click on the valid button in a particular file for use in other tasks such as changing the method of placement of experiments or the type of questions to the subject

\subsection{Part two}

A student who is required to perform the practical work submitted to him by the professor before the start of the RPW must go through the following stages:

- To have an account on the platform in advance.

$\mathrm{Be}$ aware of the period in which the experiment was predeveloped by the professor and the group in which it was placed.

- Enter the platform and reserve the appropriate time from the professors' time limit.

- Waiting for the time seized to arrive and then checking the operation submitted to him.

- Starting work by answering theoretical questions, then moving to applied work, comparing it with hypothetical answers, and concluding.

Upon completion, the student presses the right button, thus completing the process. All he has done during that period is stored in a particular location via the MySQL program to be processed after being used for this students' evaluation.

\subsection{Part three}

The laboratory, which is the place of completion of operations designed by the professor where this lab has a high-quality process design computer on LabVIEW environment, NI ELVIS

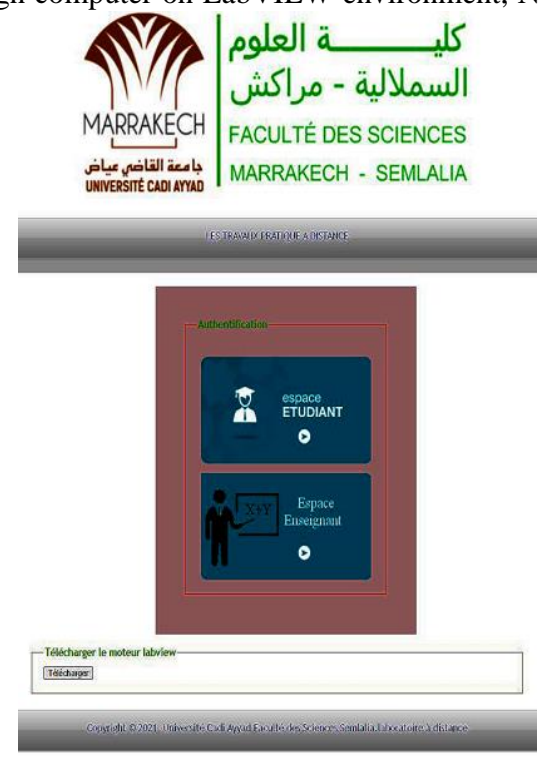

Figure 4. Home page of the platform
II+ that can realistically perform functions and link it to LabVIEW shown in figure 3 to implement what has been done in reality and add to the server that does external communication on the Internet. Also, the relay switches play an essential role in controlling the passage of electricity between the elements of the circuit to be studied.

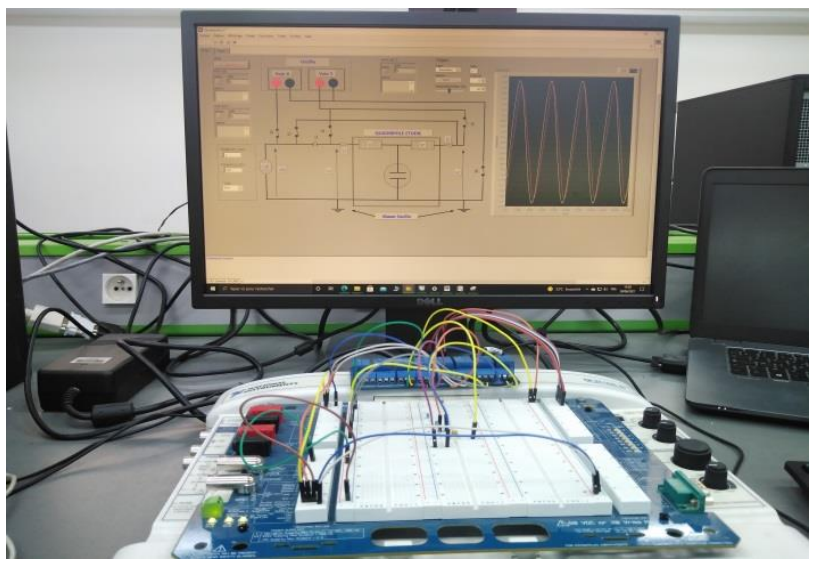

Figure 3. NI ELVIS II+ with LabVIEW environment

\section{APPLICATION}

In this section, we will try to apply what is theoretically recounted, so we will choose an experiment from the one that is used and tested in advance, and it is the cadres showing their purpose. However, there is an architecture diagrams in (Ouatik et al., 2019) explain how this application works. The objective of the manipulation is experimentally determining the impedance parameters of a linear passive quadrupole. But the real goal is to measure the degree to which students interact with the platform. In other words, has the platform been entered without problems, have the practical works been done well? What difficulties students have experienced in doing the work remotely, because that gives us feedback to develop further and repair the structure of the platform and the changes it needs to meet students' needs.

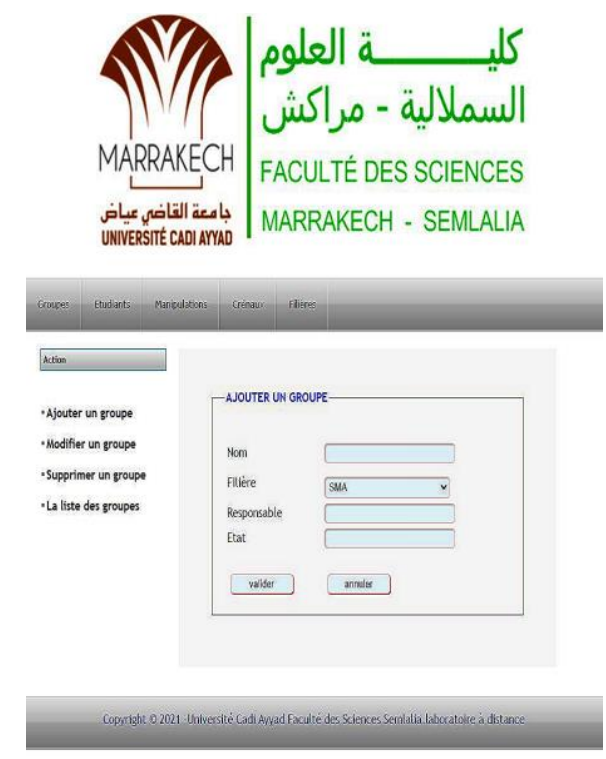

Figure 5. Teacher interface 
As shown in Figure 4, when the professor has been connecting with his account, it will be moved to the interface shown in figure 5 , which shows which icons must be entered to create and divide students into groups, add or delete students, and prepare the experience while assigning the period to each whole that has been divided.
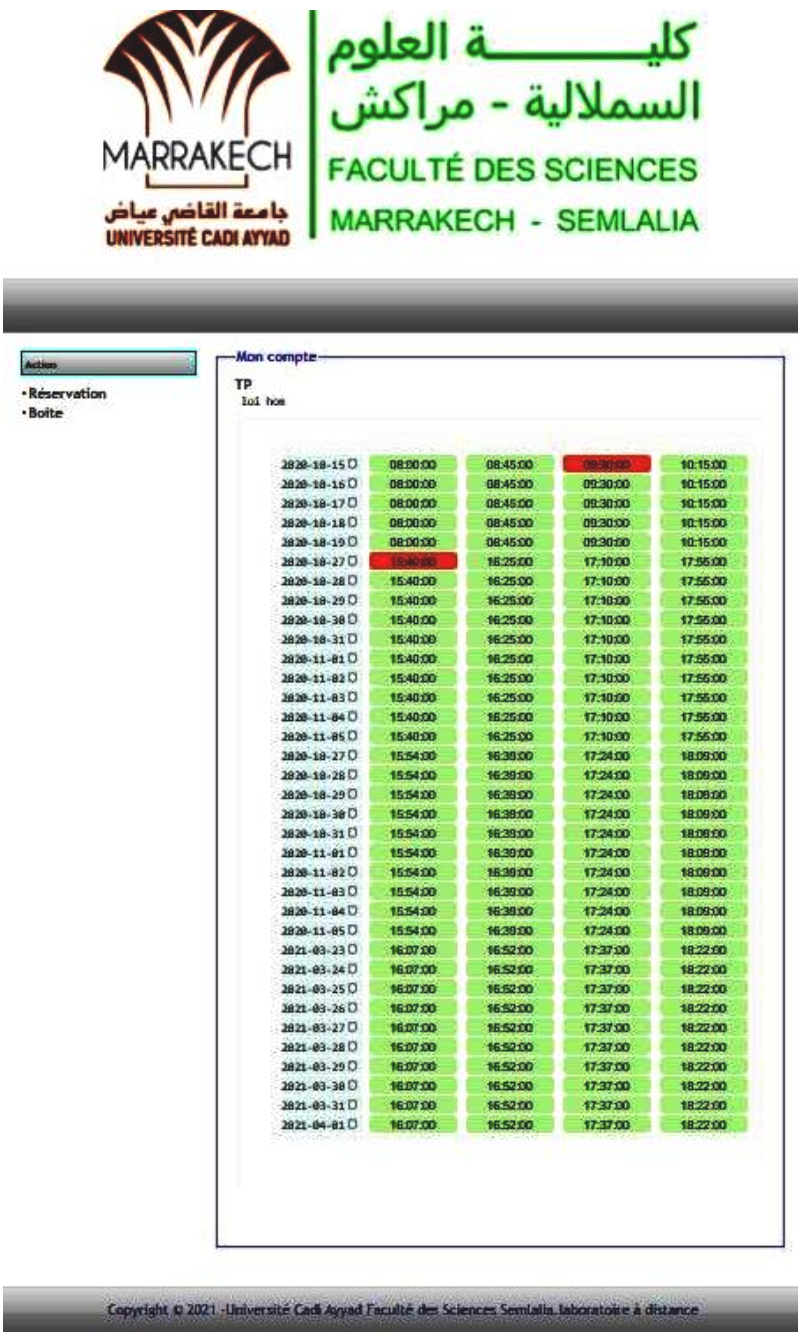

Figure 6. Student reservation interface

Figure 4 illustrates the interface that the student will be shown when entering on his icon, which will be transmitted to the interface shown in figure 6 . The student will choose the appropriate time to be reserved by the professor.

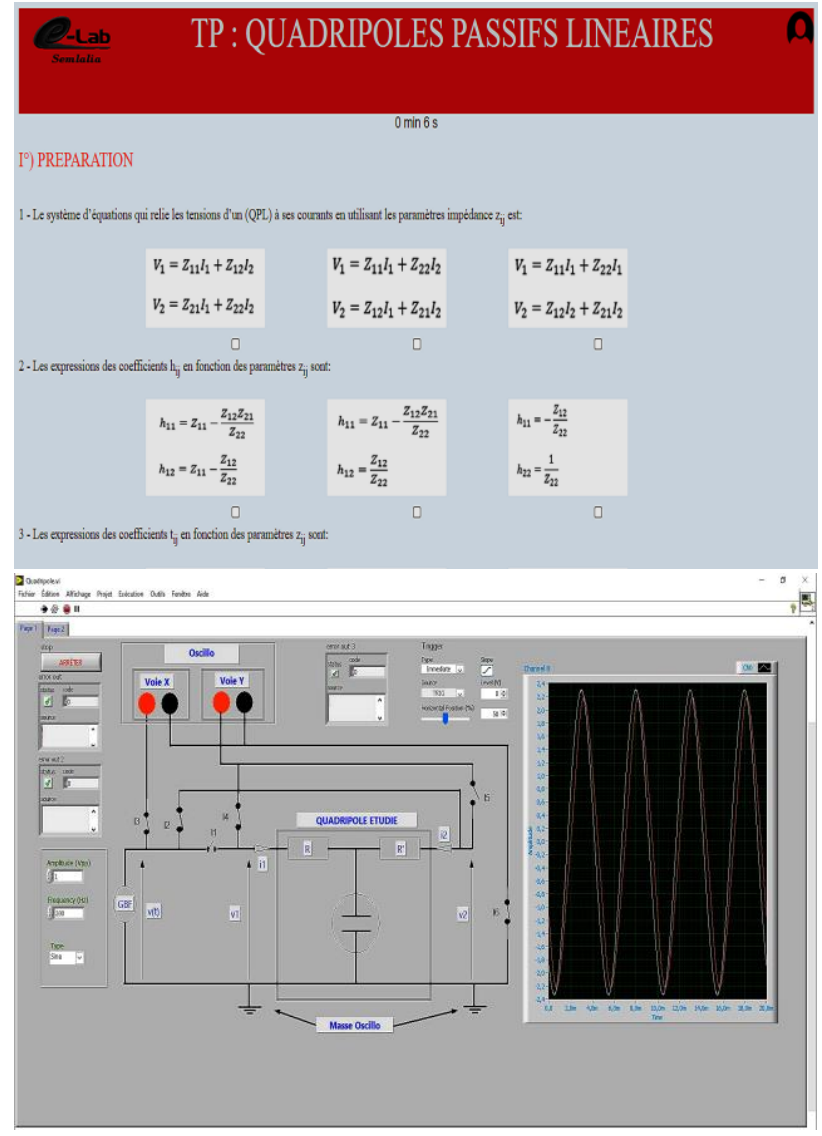

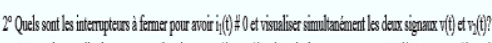

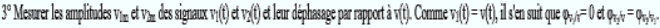

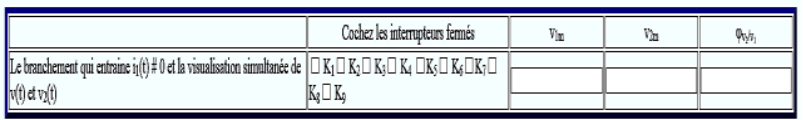

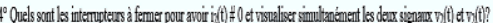

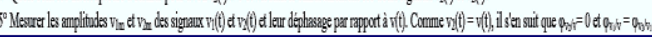

\begin{tabular}{|c|c|c|c|c|}
\hline & Cothel les intempleurs fenisis & $V_{I x}$ & $\mathrm{~F}$ & $9 \mathrm{in}$ \\
\hline 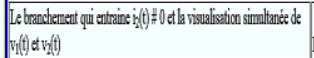 & $\begin{array}{l}\square \mathrm{K}_{1} \square \mathrm{K}_{1} \square \mathrm{K}_{j} \square \mathrm{K}_{q} \square \mathrm{K}_{j} \square \mathrm{K}_{f} \square \mathrm{K}_{j} \square \\
\mathbb{K}_{f} \square \mathrm{K}_{g}\end{array}$ & & & \\
\hline
\end{tabular}

ridur

Figure 7. Student RPW interface

Figure 7 illustrates the two-part interface of student-oriented, theoretical, multi-choice questions related to what is applied. The application part of the LabVIEW interface contains an electrical circuit for the NI ELVIS II+ board, which is added to multiplechoice questions related to an electronic circuit. After completion, the student compares two parts to the logical conclusion. 


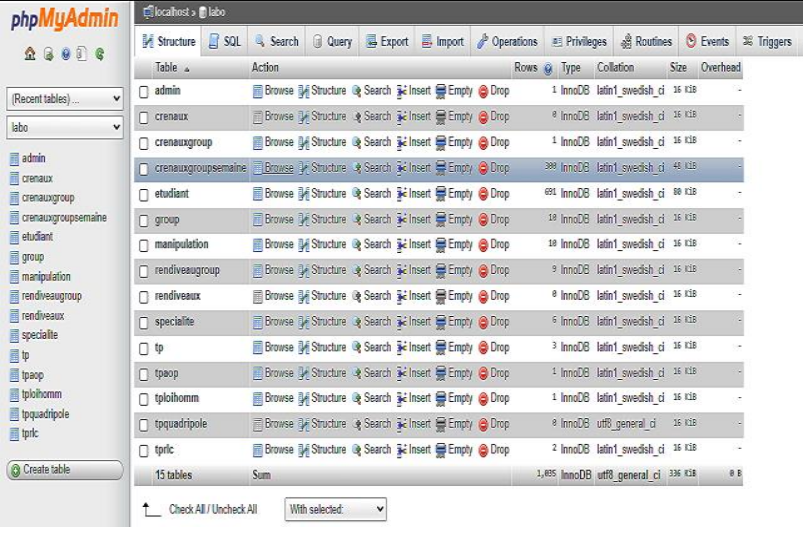

Figure 8. Student dataset storage location

After the student has finished and pressed the right button. All of his information and what has been done in detail will be stored in a particular file via the MySQL program. As shown in Figure 8 , the storage of this information and data regarding by students and professors has helped us immensely to assess students and classify them according to their abilities and profile, as well as to provide their teachers with feedback indicating the aspects to be taken into account to simplify the type of questions asked and the processes performed. Analysis and processing of student data as well as evaluation and classification of students make artificial intelligence algorithms.

\section{RESULTS AND DISCUSSION}

Our smart platform has been used as a source of data to be analysed using convolutional neural network (CNN), recurrent neural network RNN, extended short-term memory network (LSTM), and artificial neural network (ANN) techniques for data retrieval and analysis. Figure 9 shows the results that were obtained using these AI algorithms (El Gourari et al., 2022).
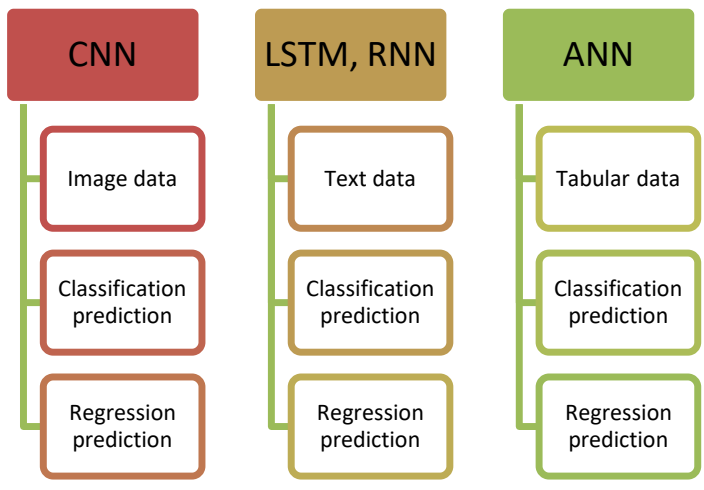

Figure 9. The AI algorithms used to solve our problem
During the activation of the experiment, 2341 records of relevant data were obtained from the educational activity of RPWs. The retrieved data records include five activities from this completed work, 30 students, and six other ready-to-use experiments. In this work, the 3 assisted experts in the field in conducting investigations and evaluating results. In our case, there is third dataset split from our original dataset which is kept hidden from training and evaluation process. The following diagram (figure 10) represents distribution data split configurations.

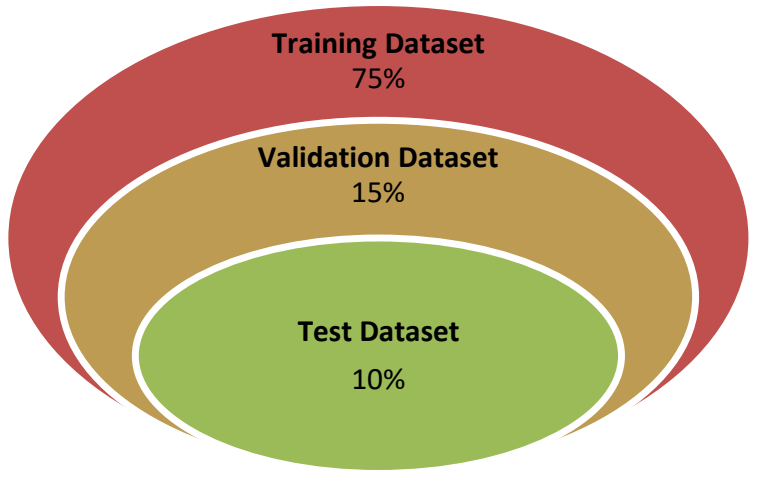

Figure 10. Visualization of the splits

We choose the Model built with training, validation, and test dataset because it has a greater likelihood of generalizing on unseen these dataset than the model built using just training dataset and the model built with training and validation dataset. The experience results from this paper show that accuracy was $41 \%$, since the accuracy of the data indicates validity and consistency. Also, it is one of the fundamental aspects of the quality of this data. That is means our data is accurate and error free. As we can conclude, these dataset have been presented in a uniform and consistent format. Student interaction with the platform was $62.21 \%$, and recall was $43.46 \%$. The experiment results proposed in the research (Ke et al., 2013) show that accuracy is $37.94 \%$, and recall is $45.71 \%$. The proposed method in this work appears to be more effective than the method proposed by (Ke et al., 2013) regarding accuracy. Still, if we compare the recall, we would find that our approach is less effective than another method. This is due to the number and type of dataset used in each method and how it is divided and processed.

These are typical predictive application in our platform based on artificial intelligence technologies:

- The professor can use the platform to receive feedback on the experience he has designed and then respond to the platform itself.

- When student participation in a particular experience is reduced, it may be helpful for a professor to ask why most students are not involved in the applied work at hand. One possible reason is that the experiments delivered during the period are ineffective. Therefore, the teacher should improve the quality of the experiment and its design to satisfy students because satisfied students are more innovative and make more effort in their studies.

- When a student is less involved in a discussion forum, content or never in range, the professor can send the student an e-mail to determine where the student finds it difficult and determine the cause of their contribution less during the experiment. The professor can also provide advice on the experience as it may help increase the students' awareness 
of productive or non-productive behaviour, which may increase participation.

- A professor can predict each students' difficulties in each evaluation and recommend relevant materials and activities to students at the next stage.

To evaluate the students, this platform helped the professor find the most relevant activities for those students, increase student participation and help students achieve high ratings. When the students' involvement in manipulation is low, the professor can redesign it to improve student interactions within their RPWs. These tools helped professors design experiences that allow students to continue to participate during evaluation.

\section{CONCLUSION}

This paper developed an intelligent online platform with many software backgrounds, such as HTML, CSS, PHP, and JavaScript. We added artificial intelligence technologies to predict and measure the degree to which students interact with the platform. Their scientific and intellectual levels also classify students. All these advantages provided by this platform will help us develop the process of doing the remote practical work well and in high quality. This will also give feedback to the professor for a better reformulation of the RPWs design method.

\section{REFERENCES}

El Gourari, A., Raoufi, M., \& Skouri, M. (2022). Formulating Quizzes Questions Using Artificial Intelligent Techniques (Mohamed Ben AhmedHoria-Nicolai L. TeodorescuTomader MazriParthasarathy SubashiniAnouar Abdelhakim Boudhir (ed.); pp. 535-547). Springer, Singapore. https://doi.org/10.1007/978-981-16-3637-0_38

El Gourari, A., Raoufi, M., Skouri, M., \& Ouatik, F. (2021). The Implementation of Deep Reinforcement Learning in E-Learning and Distance Learning: Remote Practical Work. Mobile Information Systems, 2021, 1-11. https://doi.org/10.1155/2021/9959954

El Gourari, A., Skouri, M., Raoufi, M., \& Ouatik, F. (2020). The future of the transition to e-learning and distance learning using artificial intelligence. Proceedings of the International Conference on E-Learning, ICEL, 2020-Decem, 279-284. https://doi.org/https://doi.org/10.1109/econf51404.2020.938546 4

Karadeniz, Ş. (2009). Flexible design for the future of distance learning. Procedia - Social and Behavioral Sciences, 1(1), 358363. https://doi.org/10.1016/j.sbspro.2009.01.066

Ke, C. K., Liu, K. P., \& Chen, W. C. (2013). Building a smart eportfolio platform for optimal e-learning objects acquisition. Mathematical Problems in Engineering, 2013. https://doi.org/10.1155/2013/896027

Lenar, S., Artur, F., Ullubi, S., \& Nailya, B. (2014). Problems and Decision in the Field of Distance Education. Procedia Social and Behavioral Sciences, 131(904), 111-117. https://doi.org/10.1016/j.sbspro.2014.04.088

Liang, K., Zhang, Y., He, Y., Zhou, Y., Tan, W., \& Li, X. (2017). Online Behavior Analysis-Based Student Profile for Intelligent E-Learning. Journal of Electrical and Computer Engineering, 2017. https://doi.org/10.1155/2017/9720396

Ouatik, F., Raoufi, M., Mohadab, M. El, Ouatik, F., Bouikhalene,
B., \& Skouri, M. (2019). Modeling collaborative practical work processes in an e-learning context of engineering electric education. Indonesian Journal of Electrical Engineering and Computer Science, 16(3), 1464-1473. https://doi.org/10.11591/ijeecs.v16.i3.pp1464-1473

Petrenko, L., Kravets, S., Bazeliuk, O., Maiboroda, L., \& Muzyka, I. (2020). Analysis of the current state of distance learning in the vocational education and training institutions. E3S Web of Conferences, 166. https://doi.org/10.1051/e3sconf/202016610010 66. 